OSU-HEP-09-03

May 16, 2009

\title{
New Mechanism for Neutrino Mass Generation and Triply Charged Higgs Bosons at the LHC
}

\author{
K.S. Babu1, S. Nandi2, and Zurab Tavartkiladze 3 \\ Department of Physics \\ and Oklahoma Center for High Energy Physics \\ Oklahoma State University \\ Stillwater, OK 74078, USA
}

\begin{abstract}
We propose a new mechanism for generating small neutrino masses which predicts the relation $m_{\nu} \sim v^{4} / M^{3}$, where $v$ is the electroweak scale, rather than the conventional seesaw formula $m_{\nu} \sim v^{2} / M$. Such a mass relation is obtained via effective dimension seven operators $L L H H\left(H^{\dagger} H\right) / M^{3}$, which arise when an isospin 3/2 Higgs multiplet $\Phi$ is introduced along with iso-triplet leptons. The masses of these particles are naturally in the TeV scale. The neutral member of $\Phi$ acquires an induced vacuum expectation value and generates neutrino masses, while its triply charged partner provides the smoking gun signal of this scenario. These triply charged bosons can be pair produced at the LHC and the Tevatron, with $\Phi^{+++}$decaying into $W^{+} \ell^{+} \ell^{+}$or $W^{+} W^{+} W^{+}$, possibly with displaced vertices. The leptonic decays of $\Phi^{+++}$will help discriminate between normal and inverted hierarchies of neutrino masses. This scenario also allows for raising the standard Higgs boson mass to values in excess of $500 \mathrm{GeV}$.
\end{abstract}

\footnotetext{
${ }^{1}$ E-mail: babu@okstate.edu

${ }^{2}$ E-mail: s.nandi@okstate.edu

${ }^{3}$ E-mail: zurab.tavartkiladze@okstate.edu
} 


\section{Introduction}

The existence of small neutrino masses in the range $\left(10^{-2}-10^{-1}\right) \mathrm{eV}$ has now been firmly established from a variety of neutrino oscillation experiments and serves as the only direct evidence for physics beyond the Standard Model. A question of fundamental importance is how such tiny masses, many orders of magnitude below their charged fermion counterparts, are generated. An understanding of this question will also reveal if the neutrinos are Dirac particles, very much like the charged leptons, or if they are Majorana particles, very distinct from all other fermions.

The most compelling and popular explanation is the seesaw mechanism [1] which generates neutrino masses via effective dimension five operators $L L H H / M$, where $L=(\nu, e)$ is the lepton doublet, $H=\left(H^{+}, H^{0}\right)$ is the SM Higgs doublet, and $M$ is the scale of new physics. This leads to the mass relation for light neutrinos $m_{\nu} \sim v^{2} / M$, where $\left\langle H^{0}\right\rangle=v$. These operators can arise at tree level by integrating out heavy right-handed neutrinos transforming as $(1,1,0)$ under $S U(3)_{c} \times S U(2)_{L} \times U(1)_{Y}$ (Type I seesaw), or $(1,3,2)$ Higgs bosons (Type II seesaw) [2], or $(1,3,0)$ fermions (Type III seesaw) [3], all with mass of order $M$. Neutrino oscillation data suggests $M \sim 10^{14} \mathrm{GeV}$, indicating that the associated new physics is likely to be not within reach of colliders such as the Tevatron and the LHC. While there are several indirect benefits for the seesaw, especially the one mediated by heavy right-handed neutrinos (unification of all members of a family in $S O(10)$ grand unified theory, leptogenesis), it is difficult to fathom a direct verification of the mechanism in low energy experiments. We feel that it is important to explore alternative mechanisms [4, 5, 6] which may be more directly tested [7, 8].

In this paper we propose a new mechanism for generating tiny neutrino masses at tree level via effective dimension seven operators $L L H H\left(H^{\dagger} H\right) / M^{3}$. Such operators lead to a new formula for neutrino masses, $m_{\nu} \sim v^{4} / M^{3}$, which is distinct from the standard seesaw formula. Owing to the higher dimensionality of these operators, neutrino mass generation will be more readily accessible to collider experiments in this case. Suppose that the $(1,1,0)$ and $(1,3,0)$ fermions as well as the $(1,3,2)$ Higgs bosons are not present in the fundamental theory. In this case $d=5$ neutrino mass operators will not be induced. (Such operators may still be induced by Planck scale physics, but this will lead to extremely small neutrino masses of order $10^{-5} \mathrm{eV}$, which are not relevant for neutrino oscillation data.) In such a setup, the $d=7$ operators can be the source of neutrino masses. The simplest realization of this mechanism assumes the existence of an isospin $3 / 2$ Higgs boson $\Phi=\left(\Phi^{+++}, \Phi^{++}, \Phi^{+}, \Phi^{0}\right)$ and a pair of vector-like fermions transforming as $(1,3,2)+(1,3,-2)$ under the SM gauge symmetry. $\Phi^{0}$ acquires an induced vacuum expectation value (VEV) via its interactions with the SM Higgs doublet $H$, and induces small neutrino masses. Since the mass of the $\Phi$ can naturally be in the $\mathrm{TeV}$ range, this new mass generation mechanism has interesting implications for the physics that will be explored at the LHC (and for smaller masses, at the Tevatron as well). We have analyzed the most interesting signals of this model, which occurs in 
the production and decay of triply charged Higgs boson $\Phi^{+++}$, with the possibility of displaced vertices.

\section{Model}

Our model is based on the SM symmetry group $S U(3)_{c} \times S U(2)_{L} \times U(1)_{Y}$. In addition to the usual fermions, we introduce a pair of vector-like leptons, $\Sigma=\left(\Sigma^{++}, \Sigma^{+}, \Sigma^{0}\right)$ and $\bar{\Sigma}=\left(\overline{\Sigma^{0}}, \Sigma^{-}, \Sigma^{--}\right)$ transforming as $(1,3,2)$ and $(1,3,-2)$ respectively under the gauge group. The Higgs sector consists of an isospin $3 / 2$ multiplet $\Phi=\left(\Phi^{+++}, \Phi^{++}, \Phi^{+}, \Phi^{0}\right)$ (with $Y=3$ ), in addition to the SM doublet $H=\left(H^{+}, H^{0}\right)$.

Neutrino masses arise in the model from the renormalizable Lagrangian

$$
\mathcal{L}_{\nu-\text { mass }}=Y_{i} L_{i} H^{*} \Sigma+\bar{Y}_{i} L_{i} \Phi \bar{\Sigma}+M_{\Sigma} \Sigma \bar{\Sigma}+\text { h.c. },
$$

where $Y_{i}, \bar{Y}_{i}$ are Yukawa couplings and $i$ is the family index. Integrating out the $\Sigma, \bar{\Sigma}$ fermions, one obtains an effective dimension five neutrino mass operator [6]

$$
\mathcal{L}_{\text {eff }}=-\frac{\left(Y_{i} \bar{Y}_{j}+Y_{j} \bar{Y}_{i}\right) L_{i} L_{j} H^{*} \Phi}{M_{\Sigma}}+\text { h.c. }
$$

The tree level diagram generating this operator is shown in Fig. 1. Analysis of the Higgs potential for $\Phi^{0}$ shows that it acquires an induced $\operatorname{VEV}\left\langle\Phi^{0}\right\rangle \equiv v_{\Phi}=-\lambda_{5} v^{3} / M_{\Phi}^{2}$ where $\left\langle H^{0}\right\rangle \equiv v$. When this value is substituted in Eq. (2.2), we obtain the neutrino masses to be $m_{i j}^{\nu}=\lambda_{5}\left(Y_{i} \bar{Y}_{j}+\right.$ $\left.Y_{j} \bar{Y}_{i}\right) v^{4} /\left(M_{\Sigma} M_{\Phi}^{2}\right)$. This is the dimension seven mass generation mechanism. With $\left(Y_{i}, \bar{Y}_{i}, \lambda_{5}\right) \sim$ $10^{-3}$, which are all in the domain of natural values, we obtain neutrino masses in the $\left(10^{-2}-10^{-1}\right)$ $\mathrm{eV}$ range, consistent with neutrino oscillation data, with $M_{\Sigma}$ and $M_{\Phi}$ in the TeV scale. Eq. (2.2) implies that one of the light neutrinos is massless, which is consistent with current data. This feature arises because we integrated a single pair of $\Sigma-\bar{\Sigma}$ fermions. (One can readily add more than one pair of $\Sigma-\bar{\Sigma}$ states, in which case all neutrinos will acquire masses.) Both normal hierarchy and inverted hierarchy of neutrino masses can be realized with Eq. (2.2). Note that our neutrino mass relation $m_{\nu} M^{3} \sim v^{4}$ is distinct from the traditional seesaw relation $m_{\nu} M \sim v^{2}$ (modulo dimensionless couplings). While $d=5$ neutrino masses are not induced at tree level, they do arise at 1-loop in our model via diagrams which connect two of the $H$ legs in Fig. 1 [16]. We find these finite corrections to be $\Delta m_{\nu} / m_{\nu} \sim \frac{3}{64 \pi^{2}} \frac{M^{2}}{v^{2}}$, which is $\lesssim 1$ for $M \lesssim 2 \mathrm{TeV}$. In the SUSY version of our model, the loop diagrams will be further suppressed.

Now we assert the claim that $v_{\Phi}=-\lambda_{5} v^{3} / M_{\Phi}^{2}$ used for neutrino mass estimates by analyzing the Higgs potential. It is given by

$$
\begin{aligned}
V(H, \Phi) & =\mu_{H}^{2} H^{\dagger} H+\mu_{\Phi}^{2} \Phi^{\dagger} \Phi+\frac{\lambda_{1}}{2}\left(H^{\dagger} H\right)^{2}+\frac{\lambda_{2}}{2}\left(\Phi^{\dagger} \Phi\right)^{2}+\lambda_{3}\left(H^{\dagger} H\right)\left(\Phi^{\dagger} \Phi\right) \\
& +\lambda_{4}\left(H^{\dagger} \tau_{a} H\right)\left(\Phi^{\dagger} T_{a} \Phi\right)+\left\{\lambda_{5} H^{3} \Phi^{*}+h . c\right\}
\end{aligned}
$$




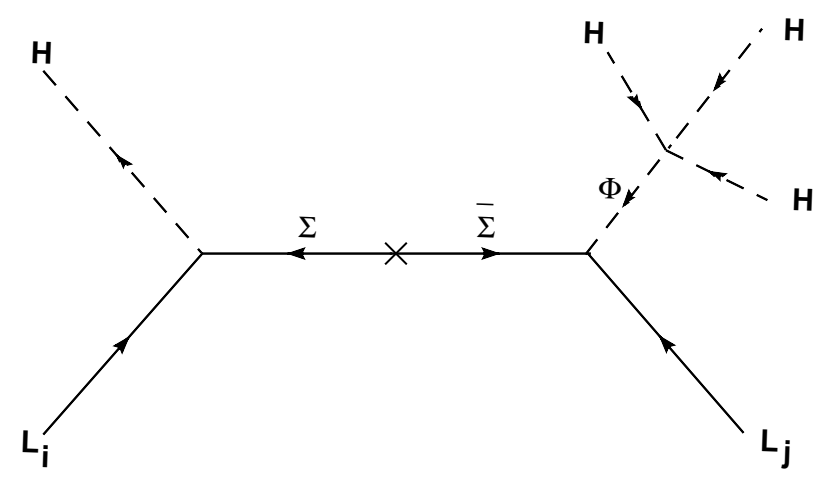

Figure 1: Tree level diagram generating dimension 7 seesaw operator for neutrino masses.

where $\tau_{a}\left(T_{a}\right)$ are the generators of $S U(2)$ in the doublet (four-plet) representation. We choose, as usual in the SM, $\mu_{H}^{2}$ to be negative, so that the vacuum breaks electroweak symmetry. $\mu_{\Phi}^{2}$ will be chosen positive, yet due to the last term in Eq. (2.3), the neutral member of $\Phi$ will acquire an induced VEV proportional to $v^{3}$. Specifically, we have

$$
v=\left(-\mu_{H}^{2} / \lambda_{1}\right)^{1 / 2}, \quad v_{\Phi}=-\lambda_{5} v^{3} / M_{\Phi}^{2}
$$

where $M_{\Phi}^{2}=\mu_{\Phi}^{2}+\lambda_{3} v^{2}+\frac{3}{4} \lambda_{4} v^{2}$ is the mass of the neutral members in $\Phi^{0}$. (These expressions ignore small corrections proportional to $v_{\Phi}^{2}$.) The mass splittings between the members of $\Phi$ are given by

$$
M_{i}^{2}=M_{\Phi}^{2}-q_{i} \frac{\lambda_{4}}{4} v^{2}
$$

where $q_{i}$ is the (non-negative) electric charge of the respective $\Phi_{i}$ field. We see that the mass splittings are equally spaced and that there are two possible mass orderings. For $\lambda_{4}$ positive, we have the ordering $M_{\Phi^{++}}<M_{\Phi^{++}}<M_{\Phi^{++}}<M_{\Phi^{0}}$, while for $\lambda_{4}$ negative, this ordering is reversed. We define a (small) splitting parameter $\Delta M^{2} \equiv\left(\lambda_{4} / 4\right) v^{2}$ which is approximately equal to $\left(2 M_{\Phi}\right)(\Delta M)$.

The important phenomenological parameters of the model are $v_{\Phi}, \Delta M, M_{\Phi}$ and $M_{\Sigma}$. In this paper, for simplicity, we shall assume that the triplet fermions $\Sigma+\bar{\Sigma}$ have masses beyond the reach of LHC, and focus on the signatures of the Higgs bosons from $\Phi$. We shall explore the entire range of $100 \mathrm{GeV}$ to $1 \mathrm{TeV}$ for $M_{\Phi}$. As discussed, the VEV $v_{\Phi}$ can naturally be in the sub-MeV range for $M_{\Phi}, M_{\Sigma}$ in the TeV range. $v_{\Phi} \neq 0$ modifies the tree-level relation for the electroweak $\rho$ parameter, which is now modified from 1 to $\rho \simeq 1-\left(6 v_{\Phi}^{2} / v^{2}\right)$. Comparing with the experimental constraint of $1.0000+0.0011(-0.0007)$ on $\rho$ [9], we obtain, at $3 \sigma$ level, $v_{\Phi}<2.5 \mathrm{GeV}$. The mass splittings between the components of $\Phi$ will induce an additional positive contribution to $\rho$, given by $\Delta \rho \simeq\left(5 \alpha_{2}\right) /(6 \pi)\left(\Delta M / m_{W}\right)^{2}[10]$. This sets an upper limit of $\Delta M<38 \mathrm{GeV}$ for the splitting 
parameter. There is also a theoretical lower limit of $\Delta M>1.4 \mathrm{GeV}$, arising from (the finite parts) of electroweak corrections [11] which we shall comply with. (This is actually a naturalness lower limit, since these corrections are not finite, with the infinity absorbed in the renormalization of the $\lambda_{4}$ coupling.) There are experimental lower limits on the masses of $\Phi, M_{\Phi}>100 \mathrm{GeV}$ [12] for a charged $\Phi$ from LEP 2, and $M_{\phi}>120 \mathrm{GeV}$ for a stable charged $\Phi$ from the Tevatron [13].

Production and decay of the $\Phi$ fields will depend on their gauge interactions. This is contained in the kinetic part of the Lagrangian for $\Phi$, given as $\mathcal{L}_{\text {kinetic }}=\left(D^{\mu} \Phi\right)^{\dagger}\left(D_{\mu} \Phi\right)$ where

$$
D_{\mu} \Phi=\left(\partial_{\mu}-i g \vec{T} \cdot \vec{W}_{\mu}-i g^{\prime} \frac{Y}{2} B_{\mu}\right) \Phi
$$

where $T_{a}$ are the $S U(2)$ generators for the isospin (3/2) representations, and $Y$ is the hypercharge.

\section{Phenomenological Implications}

Since the mass scale for the new physics responsible for light neutrino mass generation is naturally at the $\mathrm{TeV}$, our model connects neutrino physics to phenomena that can be observed at high energy colliders, such as the LHC and the Tevatron. Specially, our model has triply charged Higgs bosons which will have very distinctive phenomenology, such as displaced vertices in the detectors, and large number of $W$ 's and/or charged multi-leptons in the final states. Here we outline the decay characteristics of these bosons and their production cross sections, and the ensuing final state signals.

As noted earlier, the mass ordering inside the $\Phi$ multiplet has two options. Here we consider the case in which $\Phi^{+++}$is the lightest among the $\Phi$ 's. This is the case in which phenomenological implications of our model are most distinctive. To study the signals of these bosons, we first consider the various decay modes of $\Phi$ 's and then the production cross section at the Tevatron and the LHC.

\subsection{Decay of $\Phi$}

$\Phi^{+++}$has two principal decay modes: $\Phi^{+++} \rightarrow W^{+} W^{+} W^{+}$and $\Phi^{+++} \rightarrow W^{+} \ell^{+} \ell^{+}$. These decays arise through the diagrams where $\Phi^{+++}$emits a real $W^{+}$and an off-shell $\Phi^{++}$which subsequently decays to either two real $W^{+}$, or two same sign charged leptons. The relevant couplings are: $\Phi^{+++} \Phi^{--} W^{-}: \sqrt{3 / 2} g\left(p_{1}-p_{2}\right)_{\mu} ; \Phi^{++} W^{-} W^{-}: \sqrt{3} g^{2} v_{\Phi} ; \Phi^{++} \ell_{i}^{-} \ell_{j}^{-}: m_{i j}^{\nu} /\left(2 \sqrt{3} v_{\Phi}\right)$. The decay rates are found to be

$$
\Gamma\left(\Phi^{+++} \rightarrow 3 W\right)=\frac{3 g^{6}}{2048 \pi^{3}} \frac{v_{\Phi}^{2} M_{\Phi}^{5}}{m_{W}^{6}} I, \quad \Gamma\left(\Phi^{+++} \rightarrow W^{+} \ell^{+} \ell^{+}\right)=\frac{g^{2}}{6144 \pi^{3}} \frac{M_{\Phi} \sum_{i} m_{i}^{2}}{v_{\Phi}^{2}} J
$$

where $I, J$ are dimensionless integrals. In the limit where $M_{\Phi} \gg m_{W}$, these integrals are approximately equal to one. In Eq. (3.7), in the leptonic decay, $m_{i}$ stand for the light neutrino masses, and 


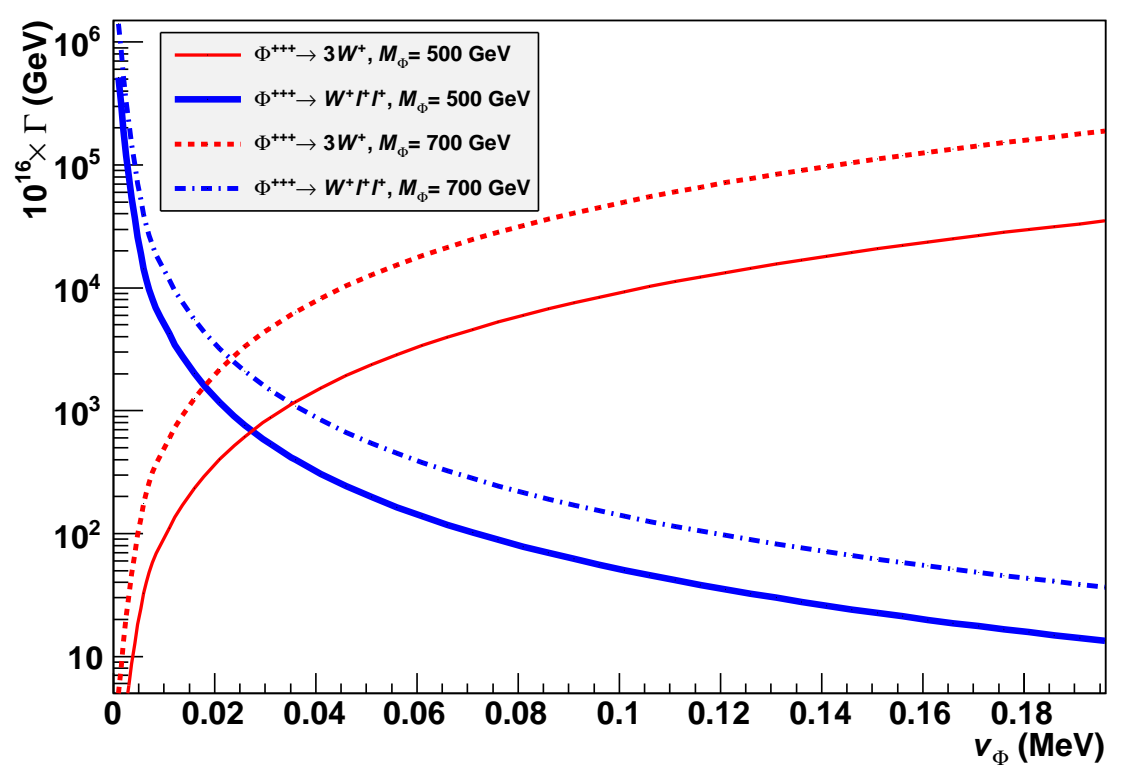

Figure 2: Decay widths $\Gamma\left(\Phi^{+++} \rightarrow 3 W^{+}\right)$(red) and $\Gamma\left(\Phi^{+++} \rightarrow W^{+} \ell^{+} \ell^{+}\right)$(blue) versus $v_{\Phi}$. Solid lines correspond to $M_{\Phi^{+++}}=500 \mathrm{GeV}$, and dashed curves to $M_{\Phi^{++}}=700 \mathrm{GeV}$.

all flavors of leptons have been summed. For our numerical evaluation we have adopted the normal hierarchy of neutrino masses with $m_{3}=0.05 \mathrm{eV}$. Results for the inverted hierarchy spectrum can be obtained by multiplying the leptonic widths by a factor of 2 .

The exact results for the partial decay widths in the $W^{+} W^{+} W^{+}$mode and $W^{+} \ell^{+} \ell^{+}$mode are shown in Fig. 2 as a function of $v_{\Phi}$ for $M_{\Phi}=500 \mathrm{GeV}$ and $700 \mathrm{GeV}$. The same is shown in Fig. 3 as a function of $M_{\Phi}$ for $v_{\Phi}=0.01 \mathrm{MeV}$ and $0.05 \mathrm{MeV}$. There are several interesting characteristics for these decays. (i) Since the $W W W$ mode is proportional to $v_{\Phi}^{2}$, while the $W^{+} \ell^{+} \ell^{+}$mode scales as $1 / v_{\Phi}^{2}$, the former is the dominant one for larger values of $v_{\Phi}$, while the latter is dominant for smaller values of $v_{\Phi}$. The two widths are equal at $v_{\Phi} \sim 0.03 \mathrm{MeV}$ for $M_{\Phi}=500 \mathrm{GeV}$. (ii) For widths $10^{-12} \mathrm{GeV}$ or smaller, $\Phi^{+++}$will travel at least $0.2 \mathrm{~mm}$, and thus will produce displaced vertex in the detector. For $M_{\Phi}=500 \mathrm{GeV}$, the width for the $W W W$ mode equals $10^{-12} \mathrm{GeV}$ for $v_{\Phi}=0.1 \mathrm{MeV}$, whereas for the $W^{+} \ell^{+} \ell^{+}$mode, this happens for $v_{\Phi}=0.005 \mathrm{MeV}$. Thus a $500 \mathrm{GeV}$ $\Phi^{+++}$will produce displaced vertex in the detector for a $v_{\Phi}$ range of $0.005-0.1 \mathrm{MeV}$. For heavier (lighter) $\Phi^{+++}$, this range in $v_{\Phi}$ is smaller (larger). A few hundred $\mathrm{GeV} \Phi^{+++}$can travel as much as a meter in the detector before it decays. (iii) If $v_{\Phi}$ is much larger $0.1 \mathrm{MeV}$, then it will decay immediately inside the detector into $W W W$.

Let us now briefly comment on the decays of the heavier $\Phi$ 's. $\Phi^{++}$has three principal decay modes: $\Phi^{++} \rightarrow W^{+} W^{+}, \ell^{+} \ell^{+}, \Phi^{+++} W^{-*}, \Phi^{+++} \pi^{-}$. The first two decay rates depend on the value of $v_{\Phi}$, while the remaining two depend crucially on the value of $\Delta M$. The final states are $W W$, two same sign charged leptons, $W W W W^{*}$ or $W W W \pi$. For the singly charged Higgs $\Phi^{+}$(which 


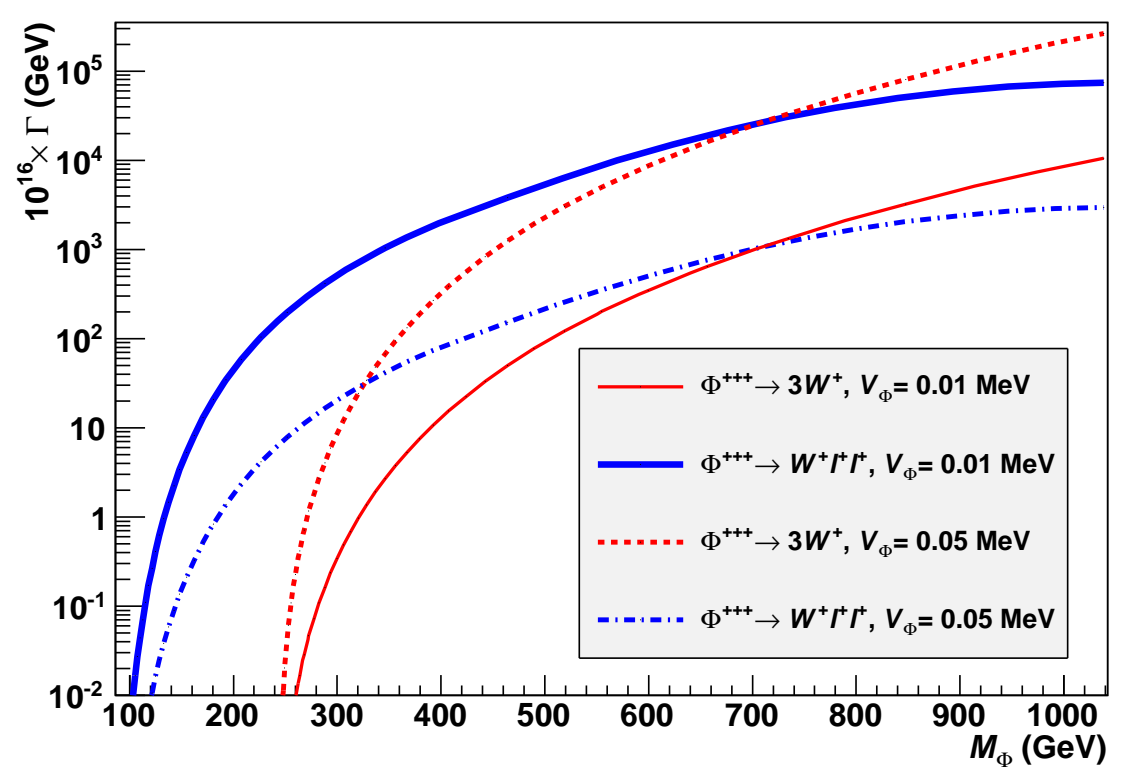

Figure 3: Decay widths $\Gamma\left(\Phi^{+++} \rightarrow 3 W^{+}\right)$(red) and $\Gamma\left(\Phi^{+++} \rightarrow W^{+} \ell^{+} \ell^{+}\right)$(blue) versus $M_{\Phi}$. Solid lines correspond to $v_{\Phi}=0.01 \mathrm{MeV}$, and dashed curves to $v_{\Phi}=0.05 \mathrm{MeV}$.

mixes weakly with the SM Higgs), decay modes are $\Phi^{+} \rightarrow \Phi^{++} W^{-*}, \Phi^{++} \pi^{-}$, with the subsequent decays of $\Phi^{++}$as above. Thus the final states will be $W W W^{*}, W W W W^{*} W^{*}, \ell^{+} \ell^{+} W^{*}$, and so on. Finally for the neutral component, $\Phi^{0}$ (which also mixes weakly with the SM Higgs), the final state decay products can have as many as six $W^{\prime}$ s $\left(W W W W^{*} W^{*} W^{*}\right)$, or a combination of $W^{\prime}$ s and charged multi-leptons.

\subsection{Production of $\Phi$}

At the Tevatron and the LHC, $\Phi^{+++} \Phi^{--}$and $\Phi^{++} \Phi^{---}$are pair produced via $s$-channel $W^{+}$ and $W^{-}$exchanges, while $\Phi^{+++} \Phi^{---}$are pair produced via the $s$-channel $\gamma$ and $Z$ exchanges. The $\Phi^{+++} \Phi^{---} Z$ coupling is $-\left(3 e \cos 2 \theta_{W} / \sin 2 \theta_{W}\right)\left(p_{1}-p_{2}\right)_{\mu}$, while the $\phi^{+++} \phi^{--} W_{\mu}^{-}$vertex is $\sqrt{3 / 2} g\left(p_{1}-p_{2}\right)_{\mu}$. The cross sections for $\Phi^{+++} \Phi^{--}$production at the LHC $(p p, \sqrt{s}=14 \mathrm{TeV})$ and Tevatron $(p \bar{p}, \sqrt{s}=2 \mathrm{TeV})$ are shown in Fig. 4 as a function of the mass. We have taken the masses of $\Phi^{+++}$and $\Phi^{--}$to be the same. (The cross section for $\Phi^{++} \Phi^{---}$production is approximately a factor of five smaller at the LHC). At LHC, for a mass of $500 \mathrm{GeV}, \Phi^{+++} \Phi^{--}$production cross section is about $5 \mathrm{fb}$, while it increase to about $60 \mathrm{fb}$ for a mass of $250 \mathrm{GeV}$. For $v_{\Phi}$ in the range of $0.005-0.1 \mathrm{MeV}$, this will produce very distinctive events with displaced vertices and five $W$ in the final state. For very small values of $v_{\Phi}$, the decay mode $\Phi^{+++} \rightarrow W^{+} \ell^{+} \ell^{+}$will dominate, with the final state being $W^{+} \ell^{+} \ell^{+} \ell^{-} \ell^{-}$, possibly with displaced vertex. Such events are not expected in the SM, and will be a clear signal for new physics. For the Tevatron, the cross sections are much smaller, however, there may still be such observable events. For example, for a $\Phi$ mass of $200 \mathrm{GeV}$, 


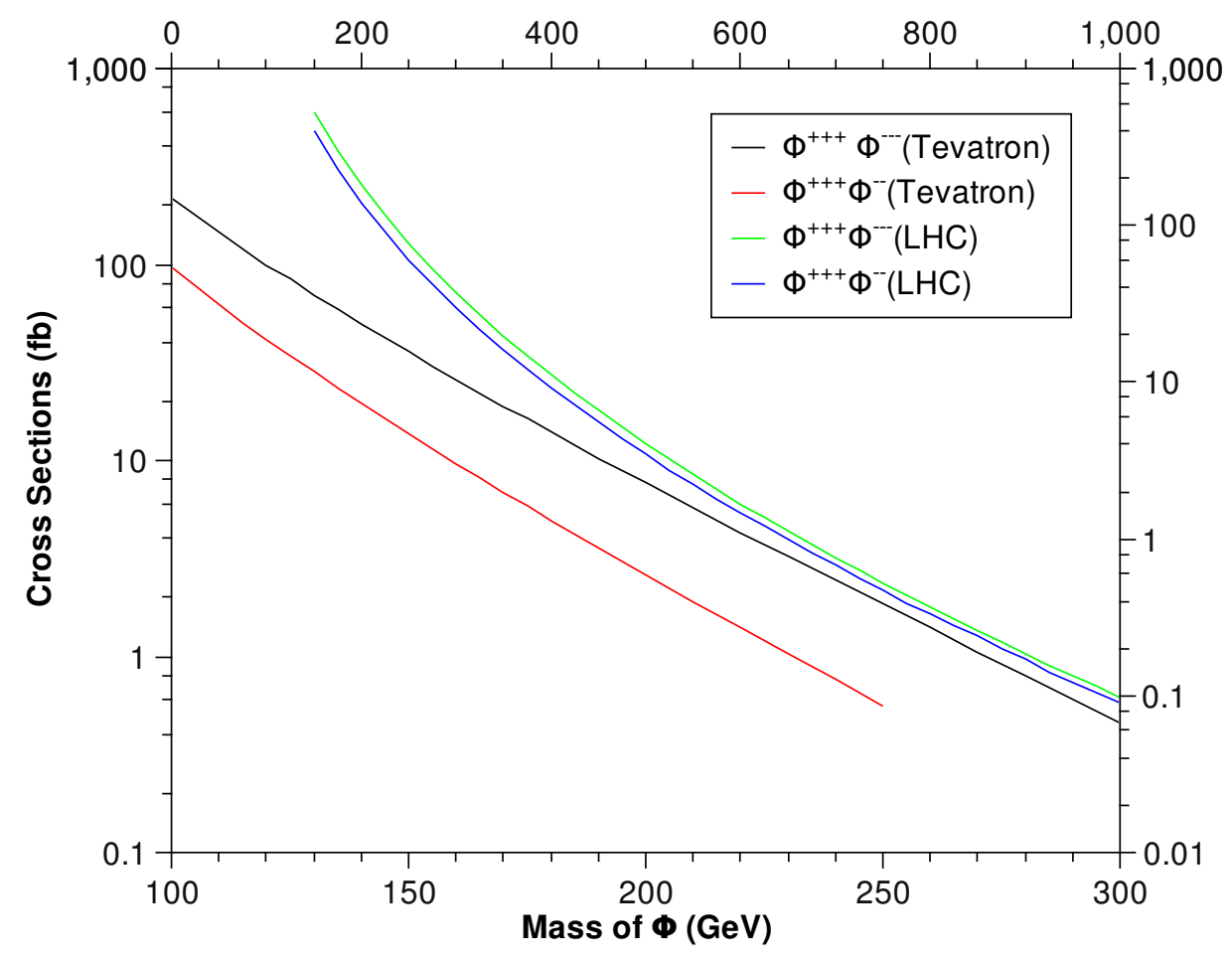

Figure 4: Cross Sections (in fb) for $\Phi$ production vs mass (in GeV) at the LHC (pp, $\sqrt{s}=14 \mathrm{TeV}$ ) and at the Tevatron $(p \bar{p}, \sqrt{s}=2 \mathrm{TeV})$. The top horizontal and the right vertical axes are for the LHC, whereas the bottom horizontal and the left vertical axes are for the Tevatron.

the cross section is about $2.6 \mathrm{fb}$ corresponding to such displaced vertex events.

The cross sections for the $\Phi^{+++} \Phi^{---}$production are also shown in Fig, 4. At the LHC, for a mass of $500 \mathrm{GeV}, \Phi^{+++} \Phi^{---}$production cross section is about $4 \mathrm{fb}$, while it increases to about $77 \mathrm{fb}$ for a mass of $250 \mathrm{GeV}$. The final states from these processes will produce $6 \mathrm{~W}$ or $2 \mathrm{~W}$ plus four charged leptons. If $v_{\Phi}$ is $\leq 0.005 \mathrm{MeV}$, the final state with $2 W$ plus four charged leptons will dominate. The SM background for such events will be negligible. For $v_{\Phi}$ in the range of $\sim 0.005-0.1 \mathrm{MeV}$, both $6 \mathrm{~W}$, and $2 W$ plus 4 charged lepton final states will compete. However, both final states will have displaced vertices very distinctive of new physics. For $v_{\Phi}>0.1 \mathrm{MeV}$, $6 \mathrm{~W}\left(W^{+} W^{+} W^{+} W^{-} W^{-} W^{-}\right)$final state will dominate. Leptonic decays of any two same-sign $W^{\prime}$ s will produce same sign charged dileptons with high $p_{T}$, with the final state having 8 high $p_{T}$ jets in addition (from the decays of the other four $W$ 's). This will serve to reduce the SM background severely, making the signal observable above the background. Thus our model can be tested in the entire $v_{\Phi}$ range. It is also possible that the lifetimes are so long that $\Phi^{+++}, \Phi^{---}$escape the detectors. This will produce two tracks in the detectors characteristic of heavy triply charged particles. If $v_{\Phi}$ is much larger, there will be no displaced vertex, but the final states with multi- $W$ and multi-leptons will be very distinctive. The cross sections for the $\Phi^{+++} \Phi^{---}$production for the Tevatron are also shown in Fig 4 . These are somewhat larger than the $\Phi^{+++} \Phi^{--}$production 
cross section. Both the D0 and the CDF experiments have looked for long-lived charged massive particles which escape their detectors. Using the CDF upper limit on the cross sections against the masses, we get a lower limit on such a long-lived $\Phi^{+++}$mass of $\sim 120 \mathrm{GeV}[13]$.

We now briefly discuss the production of some of the heavier states, and the ensuing final state signals. The cross section for $\Phi^{++} \Phi^{-}$, for the same mass, is larger by a factor $(4 / 3)$ compared to that of $\Phi^{+++} \Phi^{--}$because of slightly larger coupling, whereas the $\Phi^{++} \Phi^{--}$pair production cross section is significantly smaller than that for $\Phi^{+++} \Phi^{---}$. However, if the masses are small enough so that they are significantly produced, the final states are very distinctive from their chain decays. For example, pair production and subsequent decays lead to $\Phi^{+++} \Phi^{---} \rightarrow 6 W, \Phi^{++} \Phi^{--} \rightarrow$ $8 W, \Phi^{+} \Phi^{-} \rightarrow 10 W$, and $\Phi^{0} A^{0} \rightarrow 12 W$ ( $A^{0}$ being the neutral pseudoscalar), where some of the $W$ 's are off-shell. These are events with high charged lepton, or lepton plus jet multiplicity, all with high $p_{T}$, and are not expected in the SM.

\subsection{Other Implications}

Our model has interesting effects for the SM Higgs signals. The isospin 3/2 Higgs multiplet $\Phi$ with a tiny VEV essentially behaves like the inert Higgs [14]. SM Higgs boson $(H)$ mass can easily be raised to the range $400-600 \mathrm{GeV}$ in our model. The positive correction to the $\rho$ parameter proportional to $\Delta M$, along with small corrections to the $S$ parameter, weakens the usual bound of $185 \mathrm{GeV}$ on the $H$ mass. In this case, there is an interesting possibility, which further enhances the $\Phi$ production rate. $H$ can decay into a pair of triply charged bosons (for $M_{\Phi}$ of order $200 \mathrm{GeV}$ ). The $H$ production cross section at LHC via gluon fusion for $H$ mass of $500 \mathrm{GeV}$ is about $5 \mathrm{pb}$. With 1 inverse fb of data, there will be 5000 Higgs events. $H$ will mostly decay, as in the SM, to $2 W^{\prime}$ 's. But the branching ratio into triple charged Higgs pair is of order 10-20 percent (depending on the actual values of the quartic couplings $\lambda_{3}$ and $\lambda_{4}$ ), implying 500-1000 events with possibly displaced vertices. This will be a very unusual signal for the SM Higgs boson.

Our model also makes very interesting connections between the neutrino mass hierarchy and collider physics. If the mass of the $\Phi^{+++}<3 M_{W}$, then the decay mode $\Phi^{+++} \rightarrow W^{+} \ell^{+} \ell^{+}$will dominate, leading to final states with $e e, e \mu$ or $\mu \mu$ (along with $\tau$ 's). The dominance of $\mu \mu$ events will indicate normal hierarchy, while that of $e \mu(e e)$ will indicate inverted hierarchy corresponding to relative CP parity of the two heavier states being odd (even). Since CP symmetry is broken by the Majorana phases, these decay modes can be used to measure these phases (see the first paper of Ref. [7]). Thus high energy collider data will serve to distinguish between the models of neutrino masses and mixings. 


\section{Conclusion and Outlook}

We have presented a new mechanism for the generation of neutrino masses via dimension seven effective operators $L L H H\left(H^{\dagger} H\right) / M^{3}$. This leads to a new formula for light neutrino masses, $m_{\nu} \sim v^{4} / M^{3}$, which is distinct from the seesaw formula $m_{\nu} \sim v^{2} / M$. The scale of new physics can quite naturally be at the $\mathrm{TeV}$ scale. This microscopic theory that induces the $d=7$ operator has an isospin 3/2 Higgs multiplet containing triply charged Higgs bosons. We have analyzed the signatures of these bosons at the LHC and the Tevatron. Multi-lepton and multi $W$ final states, with the possibility of displaced vertices, should facilitate clean observation of these bosons. The leptonic decays of these particles carry information on the nature of neutrino mass hierarchy, which can be directly probed at colliders.

On a more theoretical side, the model presented here can be extrapolated from the weak scale all the way to the Planck scale without any breakdown of perturbation theory. The isospin $3 / 2$ Higgs representation can be readily embedded into the 35-dimensional representation of $S U(5)$ grand unified theory. In a forthcoming paper [15] we plan to present a more detailed analysis of the various observations made here.

\section{Acknowledgments}

We thank B. Gavela, B. Grossmann, H. Frisch, A. Khanov, A.V. Kotwal, Z. Murdock, R. Stoian and D. Zeppenfeld for helpful discussions. This work is supported in part by US Department of Energy, Grant Numbers DE-FG02-04ER41306 and DE-FG02-ER46140. Z.T. is also partially supported by GNSF grant 07_462_4-270.

\section{References}

[1] P. Minkowski, Phys. Lett. B 67 (1977) 421; M. Gell-Mann, P. Ramond and R. Slansky, in it Supergravity eds. P. van Nieuwenhuizen and D.Z. Freedman (North Holland, Amsterdam, 1979) p. 315; T. Yanagida, In Proceedings of the Workshop on the Baryon Number of the Universe and Unified Theories, Tsukuba, Japan, 13-14 Feb 1979; S. L. Glashow, NATO Adv. Study Inst. Ser. B Phys. 59687 (1979); R. N. Mohapatra and G. Senjanovic, Phys. Rev. Lett. 44912 (1980).

[2] J. Schechter and J. W. F. Valle, Phys. Rev. D 222227 (1980); G. Lazarides, Q. Shafi and C. Wetterich, Nucl. Phys. B 181, 287 (1981); R. N. Mohapatra and G. Senjanovic, Phys. Rev. D 23, 165 (1981).

[3] R. Foot, H. Lew, X. G. He and G. C. Joshi, Z. Phys. C 44, 441 (1989); E. Ma, Phys. Rev. Lett. 81, 1171 (1998). 
[4] A. Zee, Phys. Lett. B 93, 389 (1980); K. S. Babu, Phys. Lett. B 203, 132 (1988).

[5] S. Gabriel and S. Nandi, Phys. Lett. B 655, 141 (2007); P. Q. Hung, Phys. Lett. B 649, 275 (2007).

[6] Z. Tavartkiladze, Phys. Lett. B 528, 97 (2002).

[7] P. Fileviez Perez, T. Han, G. y. Huang, T. Li and K. Wang, Phys. Rev. D 78, 015018 (2008) and Phys. Rev. D 78, 071301 (2008); B. Bajc, M. Nemevsek and G. Senjanovic, Phys. Rev. D 76, 055011 (2007); A. Arhrib, B. Bajc, D. K. Ghosh, T. Han, G. Y. Huang, I. Puljak and G. Senjanovic, arXiv:0904.2390 [hep-ph]; S. Gabriel, B. Mukhopadhyaya, S. Nandi and S. K. Rai, Phys. Lett. B 669, 180 (2008); F. del Aguila, J. A. Aguilar-Saavedra and R. Pittau, JHEP 0710, 047 (2007); A. Aranda, J. Hernandez-Sanchez and P. Q. Hung, JHEP 0811, 092 (2008).

[8] E. J. Chun, K. Y. Lee and S. C. Park, Phys. Lett. B 566, 142 (2003); J. Garayoa and T. Schwetz, JHEP 0803, 009 (2008);M. Kadastik, M. Raidal and L. Rebane, Phys. Rev. D 77, 115023 (2008); A. G. Akeroyd, M. Aoki and H. Sugiyama, Phys. Rev. D 77, 075010 (2008);

W. Chao, Z. G. Si, Z. z. Xing and S. Zhou, Phys. Lett. B 666, 451 (2008).

[9] C. Amsler et al. [Particle Data Group], Phys. Lett. B 667, 1 (2008).

[10] M. B. Einhorn, D. R. T. Jones and M. J. G. Veltman, Nucl. Phys. B 191, 146 (1981).

[11] M. Cirelli, N. Fornengo and A. Strumia, Nucl. Phys. B 753, 178 (2006).

[12] LEP SUSY Working Group, LEPSUSYWG/02-05.1 (http://lepsusy.web.cern.ch/lepsusy/Welcome.htmlo).

[13] T. Aaltonen et al. [CDF Collaboration], arXiv:0902.1266 [hep-ex]; V. M. Abazov et al. [D0 Collaboration], Phys. Rev. Lett. 102, 161802 (2009).

[14] R. Barbieri, L. J. Hall and V. S. Rychkov, Phys. Rev. D 74, 015007 (2006).

[15] K.S. Babu, S. Nandi and Z. Tavartkiladze, to appear.

[16] We thank B. Gavela for raising this question. 